doi: $10.1017 /$ S1092852912000193

\title{
Clinical Updates in Neuropsychiatry - February 2011
}

First published online: February 1, 2011.

$\underline{\text { Return }}$

US FDA Approves Vilazodone for MDD

The United States Food and Drug Administration approved vilazodone (Viibryd, Trovis Pharmaceuticals) for the treatment of major depressive disorder (MDD). The recommended starting dose is $10 \mathrm{mg}$ QD for the first 7 days. The dosage can then be titrated to a maximum of $40 \mathrm{mg}$ QD.

Approval was based on two, 8-week randomized, double-blind, placebocontrolled trials in 869 adults (436 received vilazodone; 433 received placebo) between 18-70 years of age meeting Diagnostic and Statistical Manual of Mental Disorders, Fourth Edition-Revised criteria for MDD. In both studies, the Montgomery-Asberg Depression Rating Scale was used to assess all of the patients' depressive symptoms. By study endpoint, vilazodone was found to be superior to placebo in terms of improving the patients' depressive symptoms.

The most common adverse events (those occurring in greater than or equal to $5 \%$ of patients) were diarrhea, nausea, vomiting, and insomnia.

For more information on vilazodone, please consult the medication's full prescribing information (www.viibryd.com). - Christopher Naccari 